\title{
Self-similar condensation of rotating magnetized self-gravitating isothermal filaments
}

\author{
P. Hennebelle ${ }^{1,2}$ \\ ${ }^{1}$ Laboratoire de radioastronomie millimétrique, UMR 8540 du CNRS, École normale supérieure et Observatoire de Paris, \\ 24 rue Lhomond, 75231 Paris Cedex 05, France \\ 2 Department of Physics and Astronomy, Cardiff University, PO Box 913, 5 The Parade, Cardiff CF24 3YB, Wales, UK
}

Received 1 February 2002 / Accepted 11 October 2002

\begin{abstract}
Ordinary differential equations describing the self-similar collapse of a rotating, magnetized, self-gravitating and isothermal filament are derived. Explicit homologous solutions are studied with special emphasis on the bifurcation that occurs at the magnetosonic critical point. It is shown that there is a critical value for the toroidal magnetic field slope at the origin above which no bifurcation occurs, the solution remains homologous, and below which the density and the poloidal magnetic field tend to zero at large radius (envelope) whereas the toroidal magnetic field and azimuthal velocity relax towards a constant value. A series of spatial profiles of density, velocity and magnetic field, potentially useful for comparison with numerical or observational studies, is obtained numerically and discussed.
\end{abstract}

Key words. accretion, accretion disks - gravitation - hydrodynamics - magnetohydrodynamics (MHD) - ISM: clouds

\section{Introduction}

Understanding the physics of dense cores is a crucial issue for star formation. In this respect the shape of the cores is of great interest since it depends on the physics that drives their evolution.

Based on a statistical shape analysis, various studies have shown that the observational axis ratio distributions derived from a catalog of dense cores (e.g. Jijina et al. 1999) are not compatible with a distribution of oblate cores and compatible or marginally compatible with a distribution of prolate cores (David \& Verschueren 1987; Myers 1991; Ryden 1996; Curry 2002). The same conclusion is reached by Hartmann (2002) who shows that the major axis of the cores in Taurus is preferentially aligned with the filaments in which they are embedded.

Interestingly Jones et al. (2001) and Goodwin et al. (2002) have shown recently that the apparent axis ratio distribution of the dense cores is compatible with the cores being triaxial but being more oblate than prolate. However the physical origin of such structures is presently unclear and the prolate assumption appears thus to be the simplest one which is compatible with the current observations.

It is the purpose of the present study to investigate further the collapse of such elongated structures.

Send offprint requests to: P. Hennebelle, e-mail: patrick.hennebelle@ens.fr

\subsection{Prolate dense cores}

Static prolate cores have been found in various theoretical studies.

Curry (2000) has numerically obtained solutions of the Lane-Emden equations having a prolate shape. These solutions are periodic along the $z$-axis and reminiscent of the quasi periodicity of the cores sometimes observed in filaments (e.g. Dutrey et al. 1991).

Curry \& Stahler (2001) have shown that prolate cores can be solutions of the static MHD equations if the structure of the poloidal magnetic field is such that it compresses the cloud.

Finally cores permeated by an helical magnetic field can also be prolate (Tomisaka 1991; Habe et al. 1991; Fiege \& Pudritz 2000c). Fiege \& Pudritz (2000c) also show that a large sample of cores permeated by an helical magnetic field have a shape compatible with the observational axis ratio distribution and that the polarization hole effect (Fiege \& Pudritz 2000d), observed towards the densest part of the clouds, can be nicely interpreted as a purely geometrical effect due to cancellation of the contribution of the poloidal flux in the core and the toroidal flux in the envelope.

On the other hand, prolate cores can be the result of an instability in magnetized filaments as first investigated by Chandrasekar \& Fermi (1953) and recently by Nakamura et al. (1993) who show that a filamentary molecular clouds permeated by an helical field of various intensity can be instable through a combination of the gravitational and the magnetized 
sausage instability leading to the formation of prolate cores. More recently, Fiege \& Pudritz (2000a,b) extend this approach to molecular clouds confined by an external pressure.

\subsection{Aim of the paper}

In this paper we derive self-similar solutions that can describe the collapse of a magnetized, rotating, isothermal selfgravitating filament. We obtain four ordinary equations in $X$, the self-similar variable. These equations are very similar to the equations obtained by Larson and Penston for the collapse of a spherical non rotating and non magnetized cloud (Larson 1969; Penston 1969) and extensively studied by Hunter (1977), Shu (1977), Bouquet et al. (1985) and Whitworth \& Summers (1985). Our equations admit as exact solutions, some of the explicit solutions which are obtained by Aburihan et al. (2001) and by the author (Hennebelle 2001). These solutions present some important restrictions since they are unbounded and the magnetic poloidal pressure vanishes. The present study demonstrates that some of these solutions are indeed bounded, since a bifurcation arise at the magnetosonic point. Semi-analytical solutions that have a non-vanishing magnetic poloidal pressure are also obtained.

Our goal is to understand better the gravitational contraction of a filament since this process could be relevant in the context of prestellar prolate core collapse, and to obtain the spatial profiles of the fields resulting from the dynamical collapse of a magnetized filament. These profiles could then be compared with observational data and guide a full numerical simulation, although the filamentary geometry used in the present study is a significant departure from a prolate configuration.

The second section of the paper presents the formalism and discusses the physical meaning of the equations. In the third section, we discuss explicit solutions of these equations and we carefully address the question of the magnetosonic critical points. In the fourth section, we solve numerically the ordinary differential equations and discuss the physical meaning of the solutions. The fifth section concludes the paper.

\section{Self-similar solutions}

\subsection{Reduction to ordinary differential equations}

We consider the perfect MHD equations of a self-gravitating gas in cylindrical coordinates. We thus ignore the ambipolar diffusion process.

We make the usual self-similar reduction and set, in the usual notations:

$$
\begin{aligned}
X & =\frac{\varpi}{C_{\mathrm{s}}}, \\
\rho(r, t) & =\frac{1}{4 \pi G} \frac{R(X)}{t^{2}}, \\
\Phi(r, t) & =C_{\mathrm{s}}^{2} \phi(X), \\
V_{\varpi}(r, t) & =C_{\mathrm{s}} v_{\varpi}(X), \\
V_{\theta}(r, t) & =C_{\mathrm{s}} v_{\theta}(X), \\
V_{z}(r, t) & =\alpha \frac{z}{t},
\end{aligned}
$$

$$
\begin{aligned}
& B_{\theta}(r, t)=\sqrt{\mu_{0} C_{\mathrm{s}}^{2} /(4 \pi G)} \frac{b_{\theta}(X)}{t}, \\
& B_{z}(r, t)=\sqrt{\mu_{0} C_{\mathrm{s}}^{2} /(4 \pi G) \frac{b_{z}(X)}{t},}
\end{aligned}
$$

where $\alpha$ is a real number. The radial magnetic component has to vanish in order to ensure zero magnetic divergence.

The problem considered in the present study is thus axisymmetric and independent of $z$ but for the $z$-component of the velocity field. Also our analysis leaves aside the instabilities (e.g. gravitational, kink or sausage instability) that could develop and lead to a more complex evolution.

If $t<0$, these fields describe a contraction whereas they describe a rarefaction if $t>0$.

In the following, we will consider only the case $t<0$, i.e. the core contraction until the singularity formation, since during the subsequent evolution, it is expected that a disk will form, as it is the case in the numerical study of Nakamura et al. (1999), that cannot be described by the field stated by Eq. (1).

$R, \phi, v_{\varpi}, v_{\theta}, b_{\theta}$ and $b_{z}$ are dimensionless variables and are expected to be of order unity. The sound speed, $C_{\mathrm{s}}$, is about $0.2 \mathrm{~km} \mathrm{~s}^{-1}$ for a dense core having a temperature of $10 \mathrm{~K}$. Although in principle the self-similar solutions have no characteristic scales, realistic initial and boundary conditions limit the domain of validity. The characteristic spatial scale is given by $r \leq 0.01-0.1 \mathrm{pc}$, the characteristic time scale by $t \leq$ $10^{3-5}$ years and the density is $>10^{5-6} \mathrm{~cm}^{-3}$. The typical magnetic intensity is about $\simeq 10 \mu \mathrm{G}$ for a density of $\simeq 10^{3} \mathrm{~cm}^{-3}$. Then for higher densities, the relation:

$$
\frac{B}{B_{0}}=\left(\frac{\rho}{\rho_{0}}\right)^{k}
$$

where $1 / 3 \leq k \leq 1 / 2$ is theoretically (Mouschovias 1976; Scott $\&$ Black 1980) and observationally inferred (Crutcher 1999).

$B_{z}$ is the poloidal magnetic field. It tends usually to support the cloud against the gravitational collapse. $B_{\theta}$ is the toroidal magnetic component. It has not been taken into account in most of the collapse studies of a magnetized dense core. Such fields can be produced through the stretching of the poloidal magnetic field. The most important effect of the toroidal component is that it usually compresses the gas radially. It can also be a support in the axial direction.

The MHD equations lead with the definitions stated by Eqs. (1) to the following equations.

Continuity:

$-2 R-X R^{\prime}+\frac{1}{X} \partial_{X}\left(X v_{\varpi} R\right)+\alpha R=0$.

Radial momentum conservation:

$-X v_{\varpi}^{\prime}+v_{\varpi} v_{\varpi}^{\prime}-\frac{v_{\theta}^{2}}{X}=-\frac{R^{\prime}}{R}+\phi^{\prime}-\frac{b_{\theta}}{X R} \partial_{X}\left(X b_{\theta}\right)-\frac{b_{z} b_{z}^{\prime}}{R}$.

Azimuthal momentum conservation:

$-X v_{\theta}^{\prime}+v_{\varpi} v_{\theta}^{\prime}+\frac{v_{\varpi} v_{\theta}}{X}=0$

Axial momentum conservation:

$-\alpha+\alpha^{2}=0$. 
Poisson equation:

$\frac{1}{X} \partial_{X}\left(X \phi^{\prime}\right)=-R$

Azimuthal induction equation:

$-b_{\theta}-X b_{\theta}^{\prime}+\partial_{X}\left(v_{\varpi} b_{\theta}\right)+\alpha b_{\theta}=0$.

Axial induction equation:

$-b_{z}-X b_{z}^{\prime}+\frac{1}{X} \partial_{X}\left(X v_{\varpi} b_{z}\right)=0$.

Equation (6) admits the two solutions: $\alpha=0$ and $\alpha=1$, which correspond respectively to a filament static along the $z$-axis and to a filament collapsing homologously along this axis. The first configuration has been investigated by Inutsuka \& Miyama (1992) in the hydrodynamical case. In the magnetized case, a development around $X \simeq 0$ (see Eqs. (16)) implies that $b_{z}, v_{\theta}$ and $b_{\theta}$ cannot be all together different from zero which is an important restriction. Moreover, it is unlikely that a cloud will collapse in the radial direction only and not along the $z$-axis. Thus in the following, we will consider only the second case $\alpha=1$.

With $\alpha=1$, it is easily seen with Eqs. (3) and (7) that,

$\phi^{\prime}=R\left(v_{\varpi}-X\right)$.

It is also easy to see with Eqs. (3) and (9), that the density, $R$, and the axial magnetic fields, $b_{z}$, are proportional: $b_{z}=\Gamma_{z} R$, where $\Gamma_{z}$ is a real number.

With these two last equations, the system of Eqs. (3)-(9), reduces after some algebra to:

$$
\begin{aligned}
v_{\varpi}^{\prime}= & \frac{v_{\varpi}-X}{\left(v_{\varpi}-X\right)^{2}-1-b_{\theta}^{2} / R-\Gamma_{z}^{2} R} \\
& \times\left(\frac{v_{\theta}^{2}}{X}+\frac{1}{X}+R\left(v_{\varpi}-X\right)+\frac{\Gamma_{z}^{2} R}{X}-\frac{b_{\theta}^{2}}{X R}\right),
\end{aligned}
$$

$R^{\prime}=\frac{-v_{\varpi}^{\prime} R}{v_{\varpi}-X}-\frac{R}{X}$

$v_{\theta}^{\prime}=\frac{-v_{\varpi} v_{\theta}}{X\left(v_{\varpi}-X\right)}$,

$b_{\theta}^{\prime}=\frac{-v_{\varpi}^{\prime} b_{\theta}}{v_{\varpi}-X}$.

\subsection{Asymptotic behavior}

In this section we derive the asymptotic behavior of Eqs. (11)(14) in the limit $X \rightarrow-\infty$. The asymptotic behavior near the origin $X \rightarrow 0$ will be presented and discussed in some details in Sect. 3.

From Eqs. (11)-(14), it is seen that in the limit $X \rightarrow-\infty$, we have:

$$
\begin{aligned}
R(X) & \simeq A_{1} / X, \\
v_{\varpi}(X) & \simeq\left(A_{1}+\left(b_{\theta}^{\infty}\right)^{2} / A_{1}\right) \ln (|X|), \\
v_{\theta}(X) & \simeq v_{\theta}^{\infty} \exp \left(\left(A_{1}+\left(b_{\theta}^{\infty}\right)^{2} / A_{1}\right) \int^{|X|} \ln (u) / u^{2} \mathrm{~d} u\right), \\
b_{\theta}(X) & \simeq b_{\theta}^{\infty} \exp \left(-\left(A_{1}+\left(b_{\theta}^{\infty}\right)^{2} / A_{1}\right) / X\right),
\end{aligned}
$$

where $A_{1}$ is a negative real number.
Consequently at large distance, the azimuthal velocity and the toroidal magnetic component tend to constant values, whereas density (and poloidal magnetic field) decreases as $1 / X$. The radial velocity tends slowly to infinity. This is a consequence of the infinitely long filamentary geometry. Let us recall that the velocity of the Larson-Penston solutions tends to a finite value at infinity. In case of strong toroidal fields, $v_{\theta}$ and $b_{\theta}$ converge quickly to their asymptotic values.

In the limit $X \rightarrow-\infty$, we have:

$b_{z} / b_{\theta} \simeq \Gamma_{z} A_{1} b_{\theta}^{\infty} / X \rightarrow 0$ and the dominant forces are the gravitational force and the toroidal magnetic one.

\subsection{Physical discussions}

\subsubsection{Physical interpretations}

The system of Eqs. (11)-(14) has a clear physical meaning. It is very similar to the equations derived by Larson and Penston (Larson 1969; Penston 1969). As for the Larson-Penston equations, thermal pressure (second term of the right-hand side of Eq. (11)) and gravity (third term) are included. The solutions also include rotation (first term), magnetic poloidal forces (fourth term) and magnetic toroidal force (fifth term).

The system of Eqs. (11)-(14) describes the contraction (or expansion) of a filament with an homologous velocity along the $z$-axis. The gravitational force is zero along the $z$-axis which is consistent with an infinitely long filament.

In a more realistic situation, the cloud geometry and thus the the potential should evolve with time as for the solutions obtained by Lin et al. (1965) for a cold and unmagnetized cloud.

The velocity along the $z$-axis does not depend on $\varpi$, and consequently the solutions will have no clear physical meaning in the limit $\varpi \rightarrow \infty$. Asymptotic behaviors in this limit, will then not be considered in the following. Because of the homologous velocity field along the $z$-axis, the validity of the solutions is also restricted to a limited domain not too far from the $z=0$ plan, e.g. $|z| \leq$ few $\left|X_{\mathrm{c}}\right|\left(X_{\mathrm{c}}\right.$ is the position of the critical point, see next sections).

The magnetic poloidal field is proportional to density which simply means that ratio between the mass per unit length $(m=$ $\left.2 \pi \int \rho r \mathrm{~d} r\right)$ and the poloidal magnetic flux $\left(\psi=\int B_{z} r \mathrm{~d} r\right)$ is constant into the cloud. It is worth noting that $m / \psi \rightarrow \infty$ when $t \rightarrow 0$. This is due to the fact that part of the gas flows along the field line.

\subsubsection{Magnetosonic singularities}

Equations (11)-(14) become singular if: $\left(v_{\varpi}-X\right)^{2}-1-b_{\theta}^{2} / R-$ $\Gamma_{z}^{2} R \rightarrow 0$. This is the critical point already obtained in the Larson-Penston equations. The first term is the square of the fluid velocity relatively to the similarity profile, the second one is the sound speed, the two last terms represent the Alfvén velocity, $C_{\mathrm{a}}$. The physical meaning is that when the fluid velocity relatively to the similarity profile reaches the fastest velocity at which waves can travel, a singularity occurs. It is only the solutions that pass this singular point that have a physical meaning. The difference from the equations obtained by Larson and Penston is that since the magnetic field is included, the fastest 
waves are the fast MHD waves and travel with a velocity equal to: $\sqrt{C_{\mathrm{s}}^{2}+C_{\mathrm{a}}^{2}}$.

In their study, Bouquet et al. (1985) show that for any value of $\gamma$, the critical point reached by the homologous solutions is a node rather than a saddle. In the isothermal case, Whitworth $\&$ Summers (1995) (see also Bouquet 1984) were able to carry out a general study of the critical point (not restricted to the homologous solution) and show that if $\left|X_{\mathrm{c}}\right|<1$, the critical point is a saddle and the solution cannot pass it, whereas in the other case, the critical point is a node and the solutions are physical.

\section{Explicit homologous solutions and magnetosonic critical point}

\subsection{Explicit solutions: the homologous sub-Alfvénic core}

In this section, we derive explicit solutions of Eqs. (11), (12), (13) and (14). These solutions have already been obtained by the author (Hennebelle 2001) in an extended formulation of self-similarity (Eq. (63) with $\Gamma_{z}=0$ ). For completeness and clarity we rederive them here.

We look for homologous solutions and assume the following spatial dependence:

$$
\begin{aligned}
R(X) & =R_{0}, \\
v_{\varpi}(X) & =v_{\varpi, 0}^{\prime} X, \\
v_{\theta}(X) & =v_{\theta, 0}^{\prime} X, \\
b_{z}(X) & =b_{z, 0}=\Gamma_{z} R_{0}, \\
b_{\theta}(X) & =b_{\theta, 0}^{\prime} X=\Gamma_{\phi} R_{0} X
\end{aligned}
$$

where, as in Fiege \& Pudritz (2000a), $\Gamma_{z}$ and $\Gamma_{\phi}$ are respectively the poloidal and toroidal magnetic flux to mass ratio.

It is straightforward to show that Eqs. (12)-(14) require that:

$v_{\varpi, 0}^{\prime}=1 / 2$,

whereas Eq. (11) leads to:

$1 / 4+\left(v_{\theta, 0}^{\prime}\right)^{2}=R_{0} / 2+2\left(b_{\theta, 0}^{\prime}\right)^{2} / R_{0}$.

In Eq. (11) the terms independent of $X$ cancel out and lead to no new constraints, whereas the terms proportional to $X^{2}$ lead to the condition stated by Eq. (18). Consequently, the fields stated by Eqs. (16) with the constraint stated by Eq. (17) constitute the asymptotic form of the solutions of Eqs. (11)-(14) at $X \rightarrow 0$. The homologous solutions require two constraints (Eqs. (17) and (18)) and there are five parameters, which leaves three independent degrees of freedom (e.g. the poloidal and toroidal magnetic flux to mass ratio and the density).

Equation (18) has a clear physical meaning. It is simply the radial momentum conservation in the pressureless limit. The first term of the left-hand side is the square of $v_{\varpi, 0}^{\prime}$, and represents the advection term, the second one is the centrifugal force, the first term of the right-hand side is the gravitational force whereas the second one is the toroidal magnetic pinching. At this point there is no explicit poloidal support since the axial magnetic component is uniform. However, there is an implicit effect due to the critical point (see Sects. 3.2 and 3.3).

If $b_{\theta, 0}^{\prime}=0$ and $v_{\theta, 0}^{\prime}=0$, then $R_{0}=1 / 2$ and $v_{\theta, 0}^{\prime}=1 / 2$. This is very similar to the Larson-Penston solution that describes a spherical cloud having uniform density collapsing homologously, for which $R_{0}=2 / 3$ and $v_{\varpi, 0}^{\prime}=2 / 3$.

If $R_{0}<1 / 2$, small values of $b_{\theta, 0}^{\prime}$ are not allowed, whereas if $R_{0}>1 / 2$, small values of $v_{\theta, 0}^{\prime}$ are forbidden. In the first case, gravity is too weak and cannot produce the homologous collapse by itself, the condensation is induced by the toroidal pinching. In the second case, gravity is too strong to produce the homologous collapse and the cloud must be supported by the centrifugal force.

These solutions are valid until the critical point is reached and describe a sub-Alfvénic (relatively to the similarity profile) dense core contracting homologously.

\subsection{Critical point}

A detailed analysis of the critical point can be achieved for the solutions stated by Eqs. (18). This has already been investigated for the Larson-Penston solutions by Whitworth \& Summers (1985) in the isothermal case and by Bouquet et al. (1985) for any values of $\gamma$.

For the solutions stated by Eqs. (18), we have:

$$
\begin{aligned}
\left(v_{\varpi}-X\right)^{2}-1-b_{\theta}^{2} / R-\Gamma_{z}^{2} R & = \\
\left(\frac{1}{4}-\left(b_{\theta, 0}^{\prime}\right)^{2} / R_{0}\right) X^{2} & -1-\Gamma_{z}^{2} R_{0} .
\end{aligned}
$$

Thus if $-\sqrt{R_{0}} / 2<b_{\theta, 0}^{\prime}<\sqrt{R_{0}} / 2$, the critical point occurs at:

$X_{\mathrm{c}}=-\sqrt{\frac{1+\Gamma_{z}^{2} R_{0}}{1 / 4-\left(b_{\theta, 0}^{\prime}\right)^{2} / R_{0}}}$,

whereas if $\left(b_{\theta, 0}^{\prime}\right)^{2}>R_{0} / 4$, there is no singular point since the velocity relatively to the similarity profile is always smaller than the Alfvén speed.

The topological nature of the critical point can be studied by introducing a new parameter, $s$, such that:

$$
\begin{aligned}
\frac{\mathrm{d} X}{\mathrm{~d} s} & =D, \\
\frac{\mathrm{d} R}{\mathrm{~d} s} & =-R N-\frac{R}{X} D, \\
\frac{\mathrm{d} v_{\varpi}}{\mathrm{d} s} & =\left(v_{\varpi}-X\right) N, \\
\frac{\mathrm{d} v_{\theta}}{\mathrm{d} s} & =-\frac{v_{\theta} v_{\varpi}}{X\left(v_{\varpi}-X\right)} D, \\
\frac{\mathrm{d} b_{\theta}}{\mathrm{d} s} & =-b_{\theta} N
\end{aligned}
$$

where,

$N=\left(\frac{v_{\theta}^{2}}{X}+\frac{1}{X}+R\left(v_{\varpi}-X\right)+\frac{\Gamma_{z}^{2} R}{X}-\frac{b_{\theta}^{2}}{X R}\right)$,

$D=\left(v_{\varpi}-X\right)^{2}-1-b_{\theta}^{2} / R-\Gamma_{z}^{2} R$. 
The linearisation of the solutions stated by Eqs. (18) near the critical point, stated by Eq. (20) leads to a relation $\mathrm{d} \boldsymbol{Y} / \mathrm{d} s=$ $[M] \boldsymbol{Y}$, where $\boldsymbol{Y}=\left[X, R, v_{\varpi}, v_{\theta}, b_{\theta}\right]$ and where the matrix $[M]$ is given by the value of $\partial\left(\mathrm{d} \boldsymbol{Y}_{i} / \mathrm{d} s\right) / \partial \boldsymbol{Y}_{j}$ at the critical point. The matrix $[M]$ is easily calculated with Eqs. (21) and the derivative of $N$ and $D$. We have:

$\partial D / \partial \boldsymbol{Y}=\left[X_{\mathrm{c}}, \frac{b_{\theta}^{2}}{R^{2}}-\Gamma_{z}^{2},-X_{\mathrm{c}}, 0, \frac{-2 b_{\theta}^{c}}{R_{0}}\right]$,

$\partial N / \partial \boldsymbol{Y}=\left[\frac{-3 R_{\mathrm{c}}}{2}, \frac{-X_{\mathrm{c}}}{2}+\frac{\Gamma_{z}^{2}}{X_{\mathrm{c}}}+\frac{\left(b_{\theta}^{c}\right)^{2}}{X_{\mathrm{c}} R_{\mathrm{c}}^{2}}, R_{\mathrm{c}}, \frac{2 v_{\theta}^{c}}{X_{\mathrm{c}}}, \frac{-2 b_{\theta}^{c}}{X_{\mathrm{c}} R_{\mathrm{c}}}\right]$

where $R_{\mathrm{c}}, v_{\varpi}^{c}, v_{\theta}^{c}$ and $b_{\theta}^{c}$ are the values of the fields at the critical point.

The study of the eigenvalues of $[M]$ allows to determine the topological nature of the critical point. The characteristic polynômial $P$, of $[M]$ is:

$P(\lambda)=\lambda^{3}\left(\lambda^{2}-X_{\mathrm{c}} \lambda+\frac{X_{\mathrm{c}}^{2}}{4}\left(1-\frac{8\left(b_{\theta, 0}^{\prime}\right)^{4}}{R_{0}^{2}}\right)\right)=0$.

Its roots are zero (third order) and

$\lambda_{1}=\frac{1}{2} X_{\mathrm{c}}\left(1+2 \sqrt{2} \frac{\left(b_{\theta, 0}^{\prime}\right)^{2}}{R_{0}}\right)$,

$\lambda_{2}=\frac{1}{2} X_{\mathrm{c}}\left(1-2 \sqrt{2} \frac{\left(b_{\theta, 0}^{\prime}\right)^{2}}{R_{0}}\right)$.

0 is eigenvalue of $[M]$ at the third order since the vectors $\left(\partial\left(\mathrm{d} \boldsymbol{Y}_{i} / \mathrm{d} s\right) / \partial \boldsymbol{Y}\right)\left(X_{\mathrm{c}}\right)$ are linear combinations of $(\partial D / \partial \boldsymbol{Y})\left(X_{\mathrm{c}}\right)$ and $(\partial N / \partial \boldsymbol{Y})\left(\boldsymbol{X}_{\mathrm{c}}\right)($ see Eqs. $(21))$.

In the neighbourhood of the critical point, one has:

$\boldsymbol{Y}(s)=\Sigma_{i=1,5} \alpha_{i} \boldsymbol{V}_{i} \exp \left(\lambda_{i} s\right)$

where $\boldsymbol{V}_{i}$ are the eigenvectors and $\alpha_{i}$ are real numbers and where $\lambda_{3}, \lambda_{4}$ and $\lambda_{5}$ are the three vanishing eigenvalues.

Consequently, since $\lambda_{1}$ and $\lambda_{2}$ are both negative (remembering that $\left.\left(b_{\theta, 0}^{\prime}\right)^{2} / R_{0}<1 / 4\right)$, the critical points are nodes rather than saddles. The solutions are thus able to cross the critical point. It does not necessarily mean that the solutions are always physical, since after this first critical point, it could be possible that the solutions reach a second critical point through which it could be unable to pass. Only a numerical study seems capable to give an answer to this question.

From Eqs. (21), we have the relations:

$$
\begin{aligned}
\mathrm{d} v_{\theta} & =-\frac{v_{\theta}^{c} v_{\varpi}^{c}}{\left(v_{\varpi}^{c}-X_{\mathrm{c}}\right) X_{\mathrm{c}}} \mathrm{d} X, \\
\mathrm{~d} b_{\theta} & =-\frac{b_{\theta}^{c}}{v_{\varpi}^{c}-X_{\mathrm{c}}} \mathrm{d} v_{\varpi}, \\
\mathrm{d} R & =-\frac{R_{\mathrm{c}}}{v_{\varpi}^{c}-X_{\mathrm{c}}} \mathrm{d} v_{\varpi}-\frac{R_{\mathrm{c}}}{X_{\mathrm{c}}} \mathrm{d} X,
\end{aligned}
$$

where $v_{\theta}^{c}=v_{\theta}\left(X_{\mathrm{c}}\right), v_{\varpi}^{c}=v_{\varpi}\left(X_{\mathrm{c}}\right)$ and $R_{\mathrm{c}}=R\left(X_{\mathrm{c}}\right)$.

Thus, when the solutions cross the critical point, $v_{\theta}, b_{\theta}$ and $R$ vary according to these relations.

When the solution crosses the critical point, $D$ becomes positive. This leads to the condition:

$\mathrm{d} V_{\varpi}>\frac{X_{\mathrm{c}}^{2}+R_{0}^{2} \Gamma_{z}^{2}-\left(b_{\theta, 0}^{\prime}\right)^{2} X_{\mathrm{c}}^{2} / R_{0}}{X_{\mathrm{c}}^{2}+2 R_{0}^{2} \Gamma_{z}^{2}-\left(b_{\theta, 0}^{\prime}\right)^{2} X_{\mathrm{c}}^{2} / R_{0}} \mathrm{~d} X$
According to Eqs. (27) and (28), and as pointed out by Whitworth \& Summers (1985), there is a one dimensional space of possible solutions for $X<X_{\mathrm{c}}$ which are compatible with a given solution for $X>X_{\mathrm{c}}$.

\subsection{Expected behaviors}

The homologous solutions stated by Eqs. (16) and (18) allow us to anticipate some trends that we will recover numerically in the next section.

From Eq. (20), it is seen that, $\left|X_{\mathrm{c}}\right|$ increases with $\Gamma_{z} R_{0}=b_{z}$ and $b_{\theta, 0}^{\prime}$. From Eqs. (16), it is seen that the value of $v_{\varpi}\left(X_{\mathrm{c}}\right)$, $v_{\theta}\left(X_{\mathrm{c}}\right)$ and $b_{\theta}\left(X_{\mathrm{c}}\right)$ increases with $\left|X_{\mathrm{c}}\right|$, whereas $R\left(X_{\mathrm{c}}\right)$ and $b_{z}\left(X_{\mathrm{c}}\right)$ do not depend on $\left|X_{\mathrm{c}}\right|$.

Consequently, the following trends are expected:

- If the density increases, $\left|X_{\mathrm{c}}\right|$, the position of the magnetosonic point, decreases (remembering that $b_{z}=\Gamma_{z} R$ ). This is simply due to the fact that the Alfvén speed decreases and thus the velocity of the fastest waves decreases as well. With Eqs. (18), it is seen that the rotation $\left(v_{\theta, 0}^{\prime}\right)$ increases. This means that when density increases, the gravitational force increases as well and must be counterbalanced by the centrifugal force.

- If the poloidal magnetic intensity increases, $\left|X_{\mathrm{c}}\right|$ increases and the value of the radial velocity and toroidal magnetic field at this point increase as well.

Consequently, the trend is that the larger the magnetic poloidal intensity is, the larger $\left|v_{\varpi}\right|,\left|v_{\theta}\right|$ and $\left|b_{\theta}\right|$ will be in the super-Alfvénic part $\left(X<X_{\mathrm{c}}\right)$. This is simply due to the fact that the magnetic poloidal pressure leads to an effective sound speed equal to: $1+\Gamma_{z}^{2} R$ (see Eq. (11)) and that $V_{\varpi}, V_{\theta}$ and $B_{\theta}$ are proportional to the sound speed.

- If the toroidal field increases, the position of the critical point increases and the value of the radial velocity as well, which simply means that the cloud is more compressed by the toroidal pinching. As we already said, if $\left(b_{\theta, 0}^{\prime}\right)^{1 / 2}>R_{0} / 2$ there is no critical point since the velocity of the fastest waves is always greater than the velocity of the fluid relatively to the similarity profile. Rotation must increase (Eq. (18)) in order to counterbalance the toroidal pinching.

An important parameter is the value of the radial velocity at large $X$. According to Eq. (15), the radial velocity slowly diverges when $X \rightarrow-\infty$ but, as we already said, the physical meaning of the solutions is unclear in this limit. On the other hand, Eqs. (17) and (20) give for $v_{\varpi}\left(X_{\mathrm{c}}\right)$ the value $X_{\mathrm{c}} / 2 \leq-1$ (in sound speed unit). Consequently, it is expected that the typical radial velocity at few $X_{\mathrm{c}}$ should be as high as few times the value of the sound speed.

The smallest value of $\left|v_{\varpi}\left(X_{\mathrm{c}}\right)\right|$ is 1 and is reached for the unmagnetized solution. In that case $X_{\mathrm{c}}=-2$. Let us recall that for the Larson-Penston solution, $X_{\mathrm{c}}=-3$ and $v_{\mathrm{r}}\left(X_{\mathrm{c}}\right)=-2$. The present unmagnetized solutions are thus less dynamical, in the inner homologous part and in the radial direction, than the Larson-Penston solution. 


\section{Numerical results}

\subsection{Method}

In this section we investigate numerically the system of Eqs. (11)-(14). These equations are four ordinary differential equations that can be easily integrated by a fourth order RungeKutta method.

At $X=0$, the system is singular and one has to develop the solutions to first order and to start the integration at $X \neq 0$. The asymptotic development towards $X \rightarrow 0$ is given by Eqs. (16) and $v_{\varpi, 0}^{\prime}=1 / 2$ (Eq. (17)). Consequently, the number of free parameters is four $\left(R_{0}, v_{\theta, 0}^{\prime}, \Gamma_{z}\right.$ and $\left.b_{\theta, 0}^{\prime}\right)$ and there is one more free parameter when the solutions cross the critical point, leading to a total of five free parameters. It is thus difficult to explore all the parameter space and we will restrict the investigation to a limited parameter range. We start at $X=-10^{-5}$ and use a spatial step $\mathrm{d} X=-10^{-3}$.

The integration through the critical point requires some care. As we already said, the solutions for $X<X_{\mathrm{c}}$ compatible with a solution for $X>X_{\mathrm{c}}$ are not unique and are fixed by Eqs. (27) and by the conditions stated by Eq. (28). We proceed as follow. When the critical point is reached ( $D$ reverse sign), we select a value of $\mathrm{d} v_{\varpi}$ satisfying Eq. (28) and give to the other fields the values stated by Eqs. (27). In the following we restrict our study to $\mathrm{d} v_{\varpi}=0$.

We will first consider homologous cores (thus following Eq. (18)) and then relax this assumption and consider the more general case of non-homologous cores.

\subsection{Homologous cores and non homologous super-Alfvénic envelope}

The homologous solutions stated by Eqs. (16) and (18) play an important rôle since except for the geometry they are equivalent to the Larson-Penston solution that has been found to be in reasonable agreement with numerical simulations in previous studies (Larson 1969; Hunter 1977; Blottiau et al. 1988; Foster $\&$ Chevalier 1993). Moreover, these solutions are explicit and allow us to check carefully the validity of the numerical integration.

In this section we present the numerical integration of Eqs. (11)-(14) with the initial density $R_{0}=1 / 2$. This value is important since according to Eq. (18), gravity is exactly equal to the advection term and all values of $v_{\theta, 0}^{\prime}$ and $b_{\theta, 0}^{\prime}$ are allowed.

The other parameters are: $v_{\varpi, 0}^{\prime}=1 / 2$ and in order to explore a large range of parameters $b_{z}=0,0.5$ and 1 corresponding to $\Gamma_{z}=0,1$ and 2 respectively and $b_{\theta, 0}^{\prime}$ equal to $\alpha \sqrt{R_{0} / 4}$, whith $\alpha=0.1,0.5$ and 0.9. $v_{\theta, 0}^{\prime}$ is calculated according to Eqs. (18). We integrate until $X=5 X_{\mathrm{c}}$ and display the solutions as a function of $X / X_{\mathrm{c}}$ (see Eq. (20)). Thus, the bifurcation always occurs at $X / X_{\mathrm{c}}=1$.

Before presenting the numerical results, we briefly discuss the field intensities.
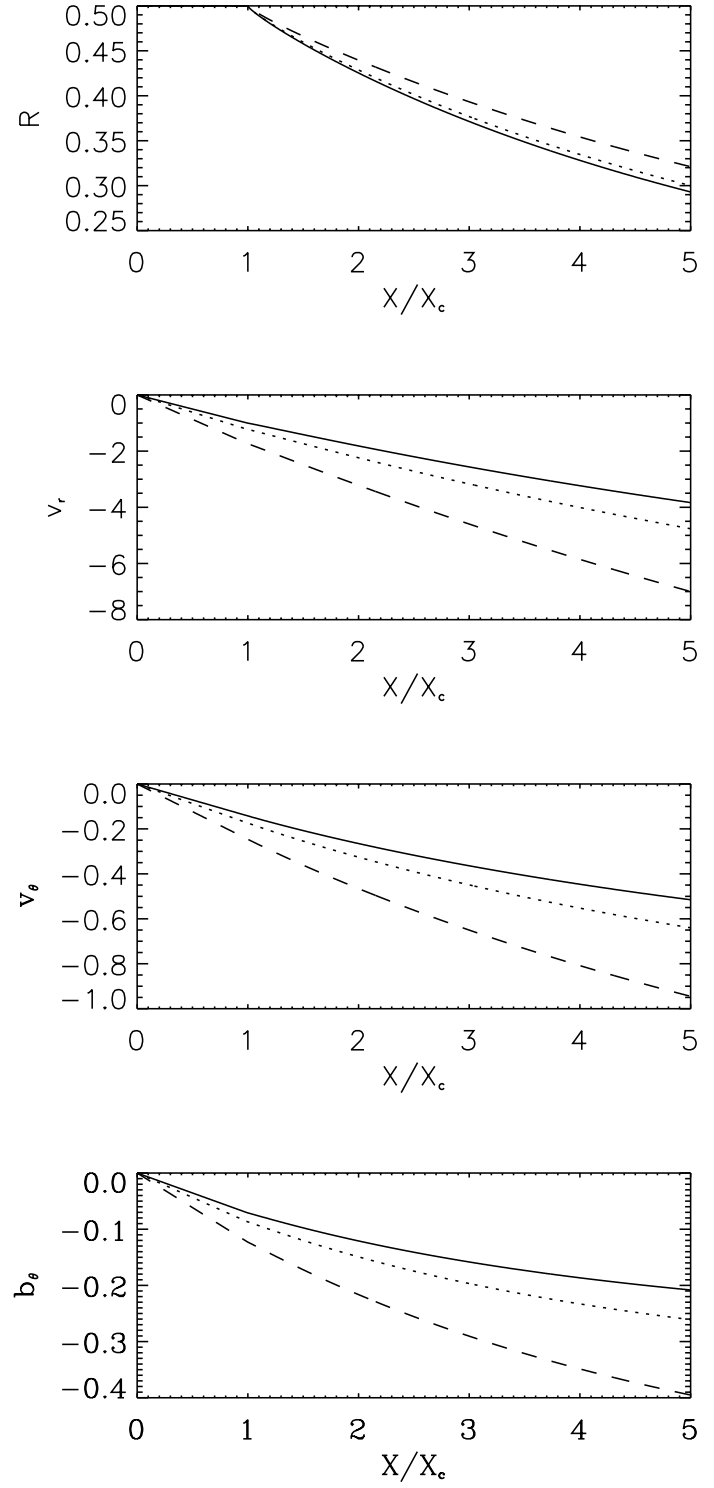

Fig. 1. Solutions corresponding to $R_{0}=1 / 2, v_{w, 0}^{\prime}=1 / 2, b_{z}=0$ (full line) 0.5 (dotted line) and 1 (dashed line) for $b_{\theta, 0}^{\prime}=0.1 \sqrt{R_{0} / 4}$. The bifurcation occurs at $X=X_{\mathrm{c}}$ according to Eq. (20).

\subsubsection{Field intensity}

For orientation, we estimate the typical values of the poloidal field in the subsonic homologous core and the value of the toroidal component at the critical point as a function of $\alpha$. With Eqs. (1)-(20), one has:

$B_{z}=\sqrt{\mu_{0} C_{\mathrm{s}}^{2} \rho} \Gamma_{z} \sqrt{R_{0}}$

$B_{\theta}=\sqrt{\mu_{0} C_{\mathrm{s}}^{2} \rho} \sqrt{\frac{\alpha^{2}}{1-\alpha^{2}}} \sqrt{1+\Gamma_{z}^{2} R_{0}}$

If a canonical density of $10^{6} \mathrm{~cm}^{-3}$ in the collapsing filament is adopted and for $R_{0}=1 / 2, \Gamma_{z}=1$, then typically in the weak, intermediate and strong toroidal field cases, toroidal field strengths of $\simeq 15, \simeq 90$ and $\simeq 320 \mu \mathrm{G}$ are associated with poloidal field of about $\simeq 90 \mu \mathrm{G}$. 

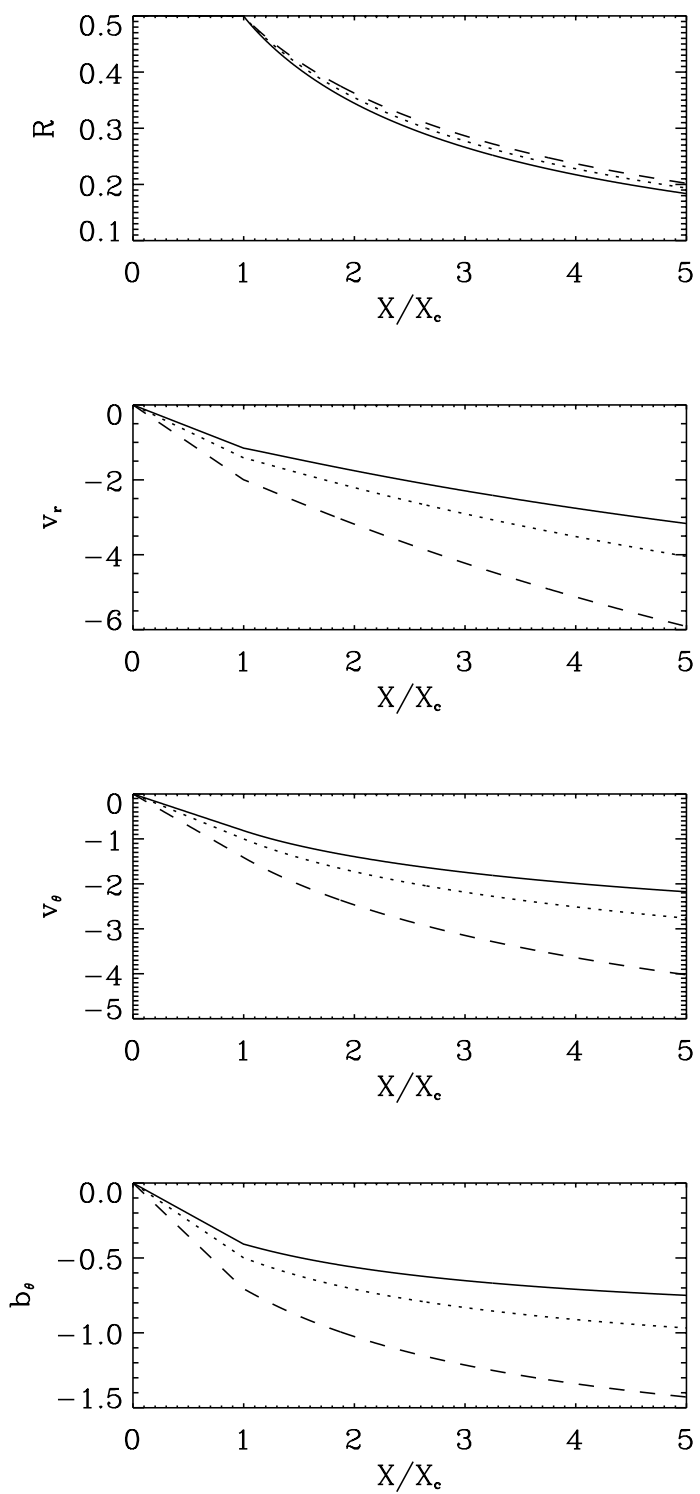

Fig. 2. Same as Fig. 1 for $b_{\theta, 0}^{\prime}=0.5 \sqrt{R_{0} / 4}$.

\subsubsection{Weak toroidal magnetic field}

Figure 1 displays the results for $b_{\theta, 0}^{\prime}=0.1 \sqrt{R_{0} / 4}$.

For $\Gamma_{z}=0$, the solution is weakly magnetized (since the toroidal field is weak) and except for the geometry it is equivalent to the Larson-Penston solution. As pointed out by Whitworth \& Summers (1985) this solution describes a strong compression wave propagating inwards into the cloud, the bifurcation point being the head of this compression wave.

It is seen that for $X / X_{\mathrm{c}}<1$ the solution is homologous as predicted by Eqs. (18). At $X / X_{\mathrm{c}}>1$ the density decreases, whereas $\left|v_{\varpi}\right|,\left|v_{\theta}\right|$ and $\left|b_{\theta}\right|$ increase. The density decreases less rapidly for large values of $b_{z}\left(\Gamma_{z}\right)$ and as discussed in Sect. 3.3, the velocity and the toroidal magnetic field increase with $b_{z}$ (see Eq. (16) and (20)).

The solutions are weakly peaked with $R(0) / R\left(5 X_{\mathrm{c}}\right) \leq 2$. The velocity and toroidal magnetic fields continue to decrease rapidly after the critical point. We have $v_{\varpi}\left(X_{\mathrm{c}}\right) / v_{\varpi}\left(5 X_{\mathrm{c}}\right) \simeq 0.25$.
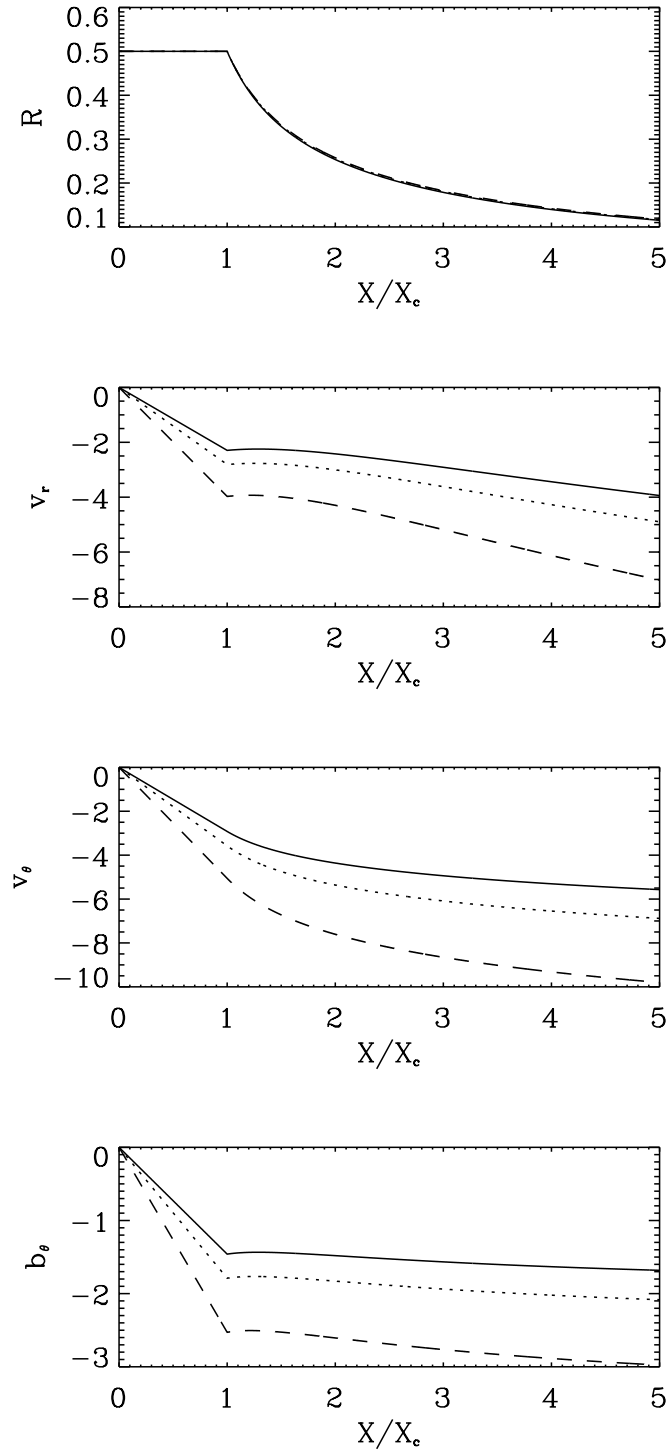

Fig. 3. Same as Fig. 1 for $b_{\theta, 0}^{\prime}=0.9 \sqrt{R_{0} / 4}$.

The radial velocity is roughly 8 times larger than the azimuthal one. When $b_{z}=0.5$ and 1 the poloidal magnetic intensity dominates the toroidal magnetic intensity in the core (by a factor $\simeq 5$ at $X_{\mathrm{c}}$ for $\Gamma_{z}=1$, and $\simeq 7$ for $\Gamma_{z}=2$ ). At $X \simeq 5 X_{\mathrm{c}}$, the two components are almost equal for $\Gamma_{z}=1$ whereas for $\Gamma_{z}=2, b_{z} / b_{\theta} \simeq 1.5$.

The radial velocity at $5 X_{\mathrm{c}}$ is around $4-5 C_{\mathrm{s}}$ for low and intermediate poloidal magnetic intensity $\left(\Gamma_{z}=0\right.$ and 1$)$ and it is much higher $(\simeq 7)$ for a strong poloidal magnetic field $\left(\Gamma_{z}=2\right)$.

\subsubsection{Intermediate toroidal magnetic field}

The case $b_{\theta, 0}^{\prime}=0.5 \sqrt{R_{0} / 4}$ is displayed in Fig. 2. The toroidal magnetic force in the core $\left(2 b_{\theta, 0}^{\prime} X / R_{0}\right)$ is about half of the gravitational force $\left(R_{0} X / 2\right)$. Thus the toroidal field is important but not dominant.

It is seen that the bifurcation at the magnetosonic point is stiffer. The density decreases more rapidly $\left(R(0) / R\left(5 X_{\mathrm{c}}\right) \leq 2.5\right)$ 
than in the previous case. This is due to the fact that the cloud is compressed by the toroidal magnetic field.

The velocity and the magnetic field evolution is much flatter in the envelope than in the core, we have $v_{\varpi}\left(X_{\mathrm{c}}\right) / v_{\varpi}\left(5 X_{\mathrm{c}}\right) \simeq 0.3$. Thus $v_{\varpi}\left(5 X_{\mathrm{c}}\right)$ is even slightly lower than for the case with weak toroidal magnetic field in spite of the higher value of $v_{\varpi}\left(X_{\mathrm{c}}\right)$.

It is also seen that the density variation with the poloidal magnetic intensity is small compared to the variation of the velocity and the toroidal magnetic component; we discuss this further in the next section.

The amplitude of the radial velocity is about $2 / 3$ the amplitude of the azimuthal one. The poloidal magnetic component dominates the toroidal one in the core (ratio 0.5-0.75 at $X=$ $X_{\mathrm{c}}$ ). The two components become comparable at $X \simeq 1.5 X_{\mathrm{c}}$ which is earlier than for the weak toroidal magnetic field. At $X / X_{\mathrm{c}}=5 b_{z} / b_{\theta} \simeq 3$.

\subsubsection{Strong toroidal magnetic field}

The case $b_{\theta, 0}^{\prime}=0.9 \sqrt{R_{0} / 4}$ is displayed in Fig. 3. The toroidal force is larger than the gravitational force (factor $\simeq 1.62$ ) and the solution describes the inward propagation of a strong magnetic compression wave into the cloud.

The most striking feature of the solutions with strong toroidal magnetic field is that the density is almost independent on the poloidal magnetic intensity, whereas the other fields are roughly proportional to $\Gamma_{z}$. This is due to the fact, that since the toroidal field is strong $\left(b_{\theta}^{2}\left(X_{\mathrm{c}}\right) / R>3\right)$, the magnetic and centrifugal forces are large compared to the thermal pressure. Thus in Eq. (11), the thermal pressure can be neglected (term -1 in $D$ and $1 / X$ in $N$ ). In this limit, it is then seen that Eq. (11) is invariant under the rescaling:

$$
\begin{aligned}
R & \rightarrow R, \\
v_{\varpi} & \rightarrow K v_{\varpi}, \\
v_{\theta} & \rightarrow K v_{\theta}, \\
b_{z} & \rightarrow K b_{z}, \\
b_{\theta} & \rightarrow K b_{\theta} .
\end{aligned}
$$

Another interesting feature is that the radial velocity and the toroidal magnetic field are almost constant just after the critical point. They then decrease significantly again around $X / X_{\mathrm{c}} \simeq$ 1.5. The radial velocity then decreases according to Eqs. (15) whereas $v_{\theta}$ and $b_{\theta}$ decrease more slowly and rapidly converge toward a constant value.

The solutions are even more peaked than in the previous cases, with $R(0) / R\left(5 X_{\mathrm{c}}\right) \simeq 5$ whereas the radial velocity is flatter with $v_{\varpi}\left(X_{\mathrm{c}}\right) / v_{\varpi}\left(5 X_{\mathrm{c}}\right) \simeq 0.5$.

The azimuthal velocity is about 1.3-1.5 larger than the radial velocity. The toroidal magnetic field rapidly dominates the poloidal one since at $X / X_{\mathrm{c}} \simeq 0.5$, the two field intensities are comparable. At $X / X_{\mathrm{c}}, b_{\theta} / b_{z} \simeq 2$ and at $X / X_{\mathrm{c}}=5$, we have $b_{\theta} / b_{z} \simeq 10$.

For $\Gamma_{z}=0$ or $\Gamma_{z}=1, v_{\varpi}\left(5 X_{\mathrm{c}}\right) \simeq 4-5 C_{\mathrm{s}}$.
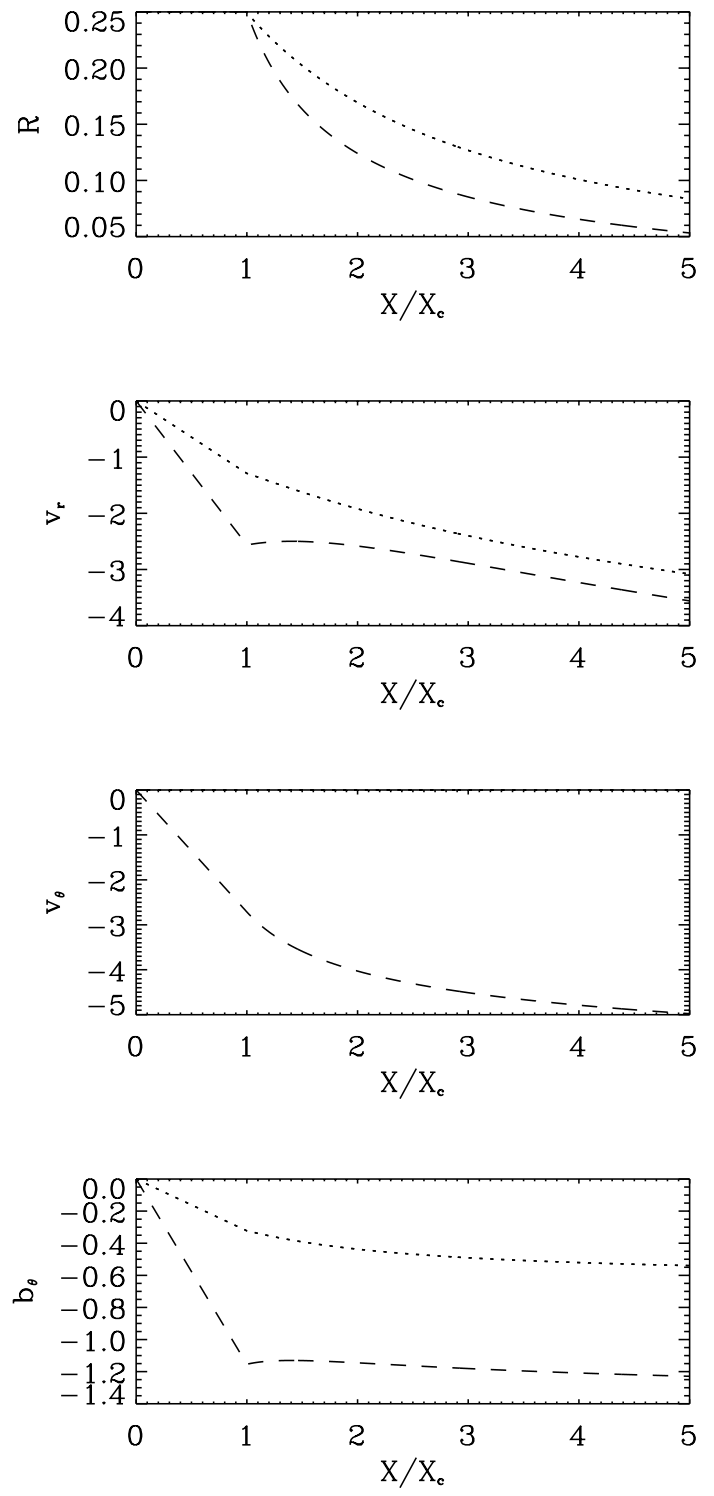

Fig. 4. Solutions corresponding to $R_{0}=0.25$ and $b_{z, 0}=0.25\left(\Gamma_{z}=1\right)$ for $b_{\theta, 0}^{\prime}=0.5 \sqrt{R_{0} / 4}$ (dotted line) and $b_{\theta, 0}^{\prime}=0.9 \sqrt{R_{0} / 4}$ (dashed line).

\subsubsection{Varying the density: Forbidden values}

In this section, we vary the density of the homologous core in order to explore the dependence of the fields on this parameter. We look carefully at two particular cases and briefly comment on other values. In order to make comparison with previous cases easier we choose $\Gamma_{z}=1$ (dotted line of previous section) and $b_{\theta}=0.1 \sqrt{R_{0} / 4}, 0.5 \sqrt{R_{0} / 4}$ and $0.9 \sqrt{R_{0} / 4}$, i.e. weak, intermediate and strong magnetic toroidal intensity.

The two cases, $R_{0}=1 / 4$ and $R_{0}=1$ are more closely considered. In the first case, gravity alone cannot explain the collapse (see Eq. (18)). The collapse is thus assisted by the toroidal magnetic field. In the second case, gravity is too strong and must be partially counterbalanced by the centrifugal force.

The results are respectively displayed in Figs. 4 and 5. The full line is $b_{\theta}=0.1 \sqrt{R_{0} / 4}$ (this case is not possible for $R_{0}=$ $1 / 4$ according to Eqs. (18)), the dotted line $b_{\theta}=0.5 \sqrt{R_{0} / 4}$ and the dashed line $b_{\theta}=0.9 \sqrt{R_{0} / 4}$. 

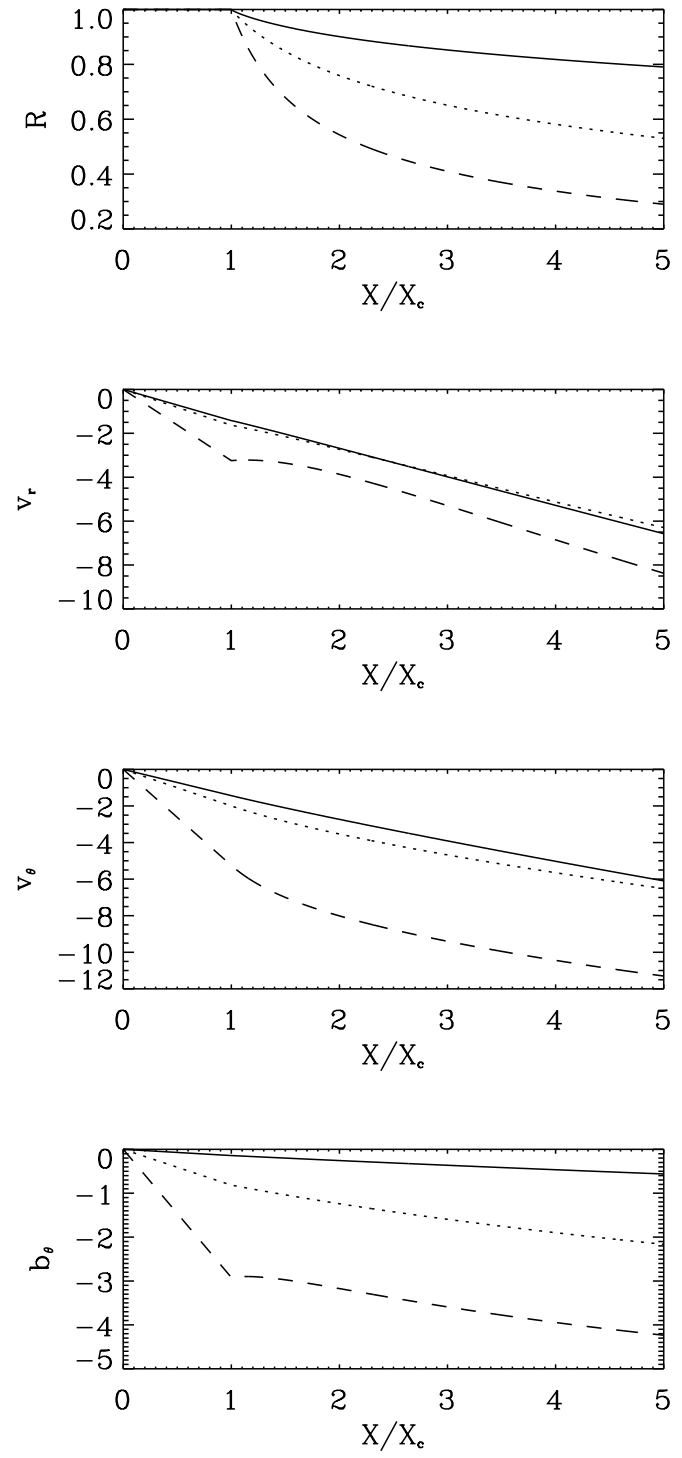

Fig. 5. Solutions corresponding to $R_{0}=1$ and $b_{z, 0}=1\left(\Gamma_{z}=1\right)$ for $b_{\theta, 0}^{\prime}=0.1 \sqrt{R_{0} / 4}$ (full line), $b_{\theta, 0}^{\prime}=0.5 \sqrt{R_{0} / 4}$ (dotted line) and $b_{\theta, 0}^{\prime}=$ $0.9 \sqrt{R_{0} / 4}$ (dashed line).

From a comparison between Figs. 4 and 5, it is seen that the density is more peaked whereas the radial velocity is flatter for small densities $\left(R_{0}=1 / 4\right)$ than for large one $\left(R_{0}=1\right)$.

For $R_{0}=1 / 4$, the azimuthal velocity vanishes according to Eq. (18) when $b_{\theta}=0.5 \sqrt{R_{0} / 4}$. When, $b_{\theta}=0.9 \sqrt{R_{0} / 4}$, it is seen that the radial velocity and the toroidal field increase slightly just after the critical point, then reduce at $X / X_{\mathrm{c}} \simeq 1.5$.

Smaller values of density $\left(R_{0}<1 / 4\right)$, require values of $b_{\theta, 0}^{\prime}$ closer to $\sqrt{R_{0} / 4}$ in order to have positive values of $\left|v_{\theta}\right|$ (see Eqs. (18)). Similar behaviors to the previously presented cases are obtained.

For larger values of density $\left(R_{0}>1\right)$, we find that with $b_{\theta, 0}^{\prime}=0$, the solutions oscillate around the homologous solution, i.e. after the critical point the density decreases and increases alternately. When $b_{\theta, 0}^{\prime} \neq 0$ (e.g. $\left.b_{\theta, 0}^{\prime}>0.1\right)$ then the solutions cross another critical point and we stop the numerical integration. This behavior is somehow similar to the results obtained by Whitworth \& Summers (1985) who find that the
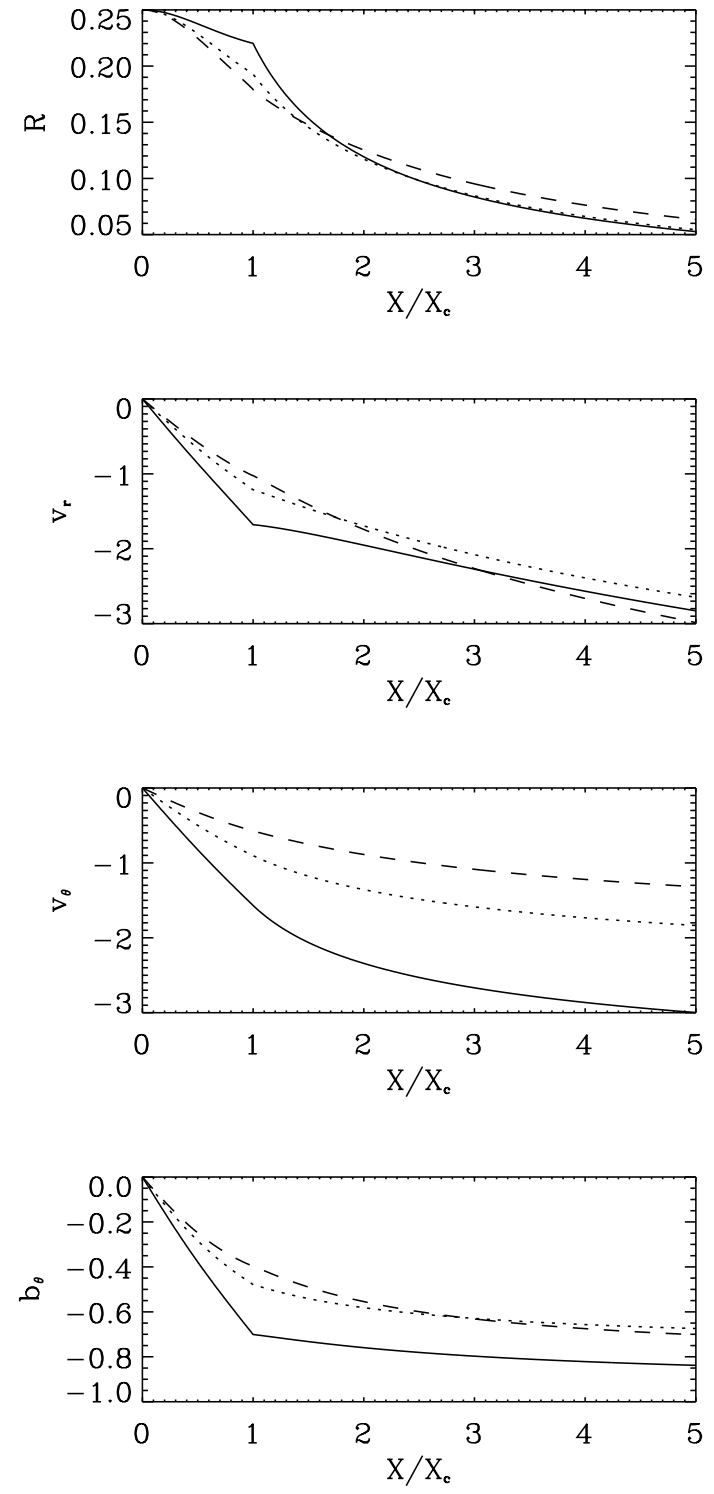

Fig. 6. Solutions corresponding to $R_{0}=0.25, b_{z, 0}=0.25, b_{\theta, 0}^{\prime}=$ $0.9 \sqrt{R_{0} / 4}$. Full line is $\delta v_{\theta}^{\prime}=-0.05$, dotted line is $\delta v_{\theta}^{\prime}=-0.15$ and dashed line $\delta v_{\theta}^{\prime}=-0.25$ (see text).

allowed values of the density at the origin in the LarsonPenston equations are quantized. It could thus be possible that larger values of $R_{0}$ are allowed in Eqs. (11)-(14). Only a systematic numerical search could answer this question.

\subsection{Non-homologous cores}

In this section, we investigate the case of non homologous cores, i.e. we start with initial conditions that do not satisfy the second conditions stated by Eqs. (18). Let us introduce the parameter, $\delta v_{\theta}^{\prime}=v_{\theta}^{\prime}-v_{\theta, 0}^{\prime}$, where $v_{\theta, 0}^{\prime}$ is the value stated by Eqs. (18). The smaller $\left|\delta v_{\theta}^{\prime}\right|$ is, the closer we are to the homologous solutions.

We consider the two cases $R_{0}=0.25$; and $R_{0}=0.5$ with $\Gamma_{z}=1$ and $b_{\theta, 0}^{\prime}=0.9 \sqrt{R_{0} / 4}$ for three values of $\delta v_{\theta}^{\prime}$ $\left(-0.05,-0.15,-0.25\right.$ for $R_{0}=0.25$ and $-0.02,-0.06,-0.12$ for $R_{0}=0.5$ ). The results are displayed in Figs. 6 and 7 . 

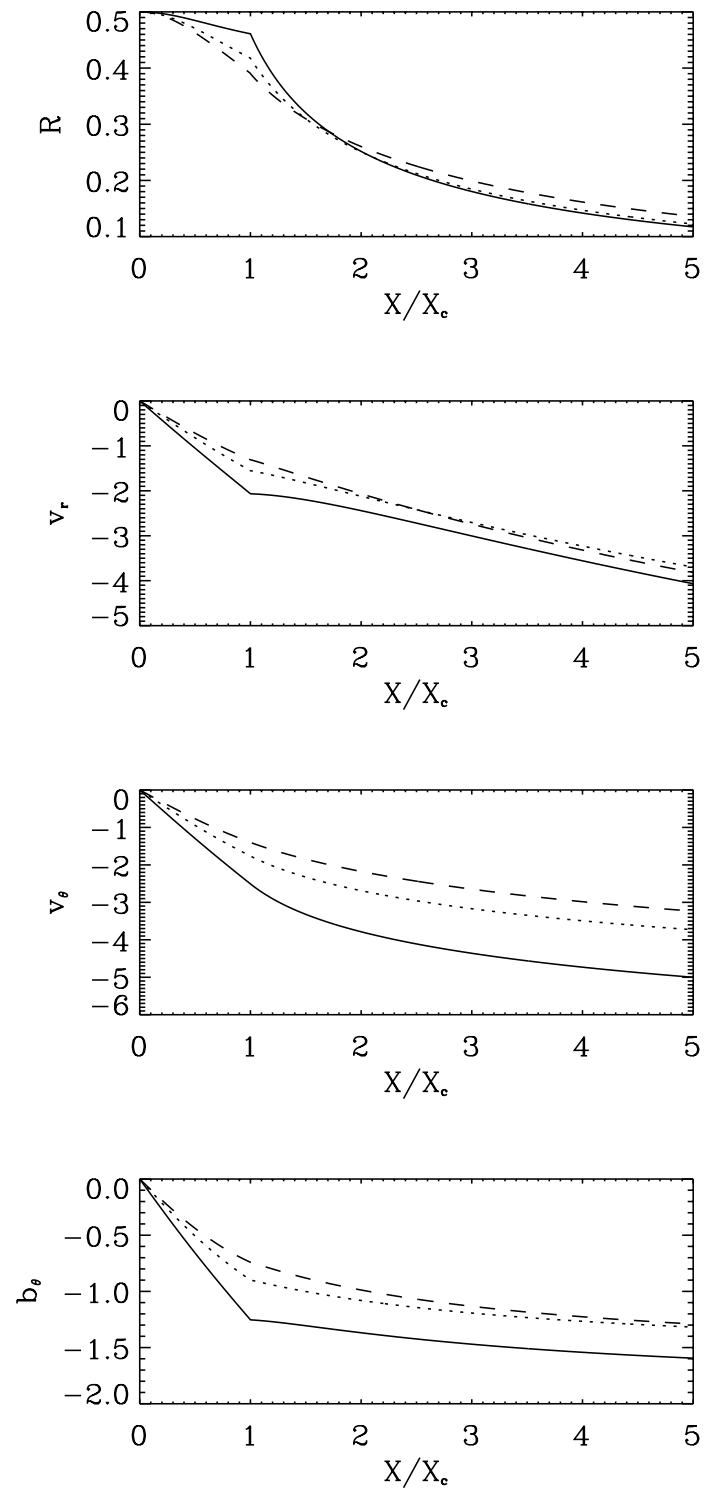

Fig. 7. Solutions corresponding to $R_{0}=0.5, b_{z, 0}=0.5, b_{\theta, 0}^{\prime}=$ $0.9 \sqrt{R_{0} / 4}$. Full line is $\delta v_{\theta}^{\prime}=-0.02$, dotted line is $\delta v_{\theta}^{\prime}=-0.06$ and dashed line $\delta v_{\theta}^{\prime}=-0.1$ (see text).

Full lines represent the smallest value of $\left|\delta v_{\theta}^{\prime}\right|$, dotted line, the intermediate value, and dashed line the largest.

It is seen that the cores are no longer homologous and become less flat when $\left|\delta v_{\theta}^{\prime}\right|$ increases. Also, the transition between the sub-Alfvénic part and the super-Alfvénic one, is smoother. The poloidal force does not vanish any more in the cores and acts explicitly everywhere.

For $R_{0}=0.25$, the density and radial velocity values at $5 X_{\mathrm{c}}$ do not vary significantly with $\delta v_{\theta}^{\prime}$, whereas the values of the azimuthal velocity and the toroidal magnetic field decrease significantly when $\left|\delta v_{\theta}^{\prime}\right|$ increases.

For larger values of $\left|\delta v_{\varpi}^{\prime}\right|$ the solutions (for $R_{0}=0.25$ and $R_{0}=0.5$ ) cross another critical point. We consider them as not physical and we stop the numerical integration. It is then not possible for these two cases to obtain solutions with a vanishing azimuthal velocity as suggested by Eq. (18).
For larger values of $R_{0}$, the largest allowed value of $\delta v_{\varpi}^{\prime}$ decreases rapidly (for $R_{0}=1$, we find that the largest allowed $\left|\delta v_{\theta}^{\prime}\right|$ is between 0.03 and 0.04 ). This is consistent with the fact that the homologous solutions with large $R_{0}$ are not physical (see Sect. 4.2.5).

It is worth noting that the value of the radial velocity at $5 X_{\mathrm{c}}$ is around 2.5 to 3 which is lower than the values obtained previously. The smallest value of $v_{\varpi}\left(5 X_{\mathrm{c}}\right)$ we found is around 2 and is obtained for $R_{0}<1 / 8, b_{\theta} \simeq \sqrt{R_{0} / 4}, \Gamma_{z}<1$ and $\left|v_{\theta, 0}^{\prime}\right|<0.2$.

This value is still 3 to 4 times higher than the value observed by Tafalla et al. (1998) in the starless cores L1544. However, it is known from previous study (e.g. Foster \& Chevalier 1993) that the Larson-Penston solution (in which the velocity tends to $3.3 C_{\mathrm{S}}$ at large $r$ ) is in good agreement with the inner collapsing part of the cloud, the external part being significantly different. This is due to the fact that the self-similar solutions make the assumption that the system is infinite and is self-similar everywhere. In particular, the self-similar assumption does not take into account the external medium. Selfsimilar solutions thus appear in the inner part of the cloud when the boundary conditions have been forgotten by the system.

\section{Conclusion}

In this paper, we have derived self-similar solutions able to describe the inner part of a collapsing, rotating, magnetized, self-gravitating, isothermal filament. The filament is collapsing homologously along the $z$-axis, the slope of the axial velocity being two times the slope of the radial one at the origin. The four ordinary equations obtained are similar to the equations derived by Penston (1969) and Larson (1969). They present a critical magnetosonic point that induces a bifurcation.

For some of the homologous solutions already obtained previously (Aburihan et al. 2001; Hennebelle 2001), we carried out a study of the critical point and found that there is a critical value of $b_{\theta, 0}^{\prime}$ (slope of the toroidal field at the origin) above which no bifurcation occurs. By studying the eigenvalues of the linearized equations in the neighbourhood of the critical point, we demonstrate that these solutions are able to cross the singularity since it is a node rather than a saddle point.

We have then explored the system numerically and obtained a series of density, velocity and magnetic fields profiles that could help to understand the observational data and could be used as benchmarks for a full numerical simulation.

The solutions obtained in this paper have some restrictions. First, they are valid not too far from the equatorial plane since they have an homologous axial velocity field that quickly diverges and not too far (few $\left.\left|X_{\mathrm{c}}\right|\right)$ from the $z$-axis since the axial component of the velocity field does not depend on $\varpi$.

Nevertheless, these solutions are the first semi-analytical solutions describing the condensation of a magnetized rotating filament and having complex (non homologous) spatial profiles. They can be used for future analytical or numerical studies of the gravo-magnetic condensation.

Acknowledgements. I thank Anthony Whitworth and Derek WardThompson for stimulating discussions. I thank D. Moss, the referee, 
for his help in improving the manuscript. I gratefully acknowledge the support of an European Commission Research Training Network under the Fifth Framework Programme (No. HPRN-CT2000-00155).

\section{References}

Aburihan, M., Fiege, J., Henriksen, R., \& Levy, T. 2001, MNRAS, 326, 1217

Blottiau, P., Bouquet, S., \& Chièze, J. P. 1988, A\&A, 207, 24

Bouquet, S. 1984, Ph.D. dissertation, Université d'Orléans Bouquet, S., Feix, M., \& Fijalkow, E. 1985, ApJ, 293, 494 Chandrasekhar, S., \& Fermi, E. 1953, ApJ, 118, 116

Crutcher, R. M. 1999, ApJ, 520, 706

Curry, C. 2000, ApJ, 541, 831

Curry, C. 2002, ApJ, 576, 849

Curry, C., \& Stahler, S. 2001, ApJ, 555, 160

David, M., \& Verschueren, W. 1987, A\&A, 186, 295

Dutrey, A., Duvert, G., Castets, A., et al. 1991, A\&A, 247, L9

Fiege, J., \& Pudritz, R. 2000a, MNRAS, 311, 85

Fiege, J., \& Pudritz, R. 2000b, MNRAS, 311, 105

Fiege, J., \& Pudritz, R. 2000c, ApJ, 534, 291

Fiege, J., \& Pudritz, R. 2000d, ApJ, 554, 830

Foster, P., \& Chevalier, R. 1993, ApJ, 416, 303
Goodwin, S., Ward-Thompson, D., \& Whitworth, A. 2002, MNRAS, 330, 769

Habe, A., Uchida, Y., Ikeuchi, S., \& Pudritz, R. 1991, PASJ, 43, 703

Hartmann, L. 2002, ApJ, 578, 914

Hennebelle, P. 2001, A\&A, 378, 214

Hunter, C. 1977 , ApJ, 218, 834

Inutsuka, S., \& Miyama, S. 1992, ApJ, 388, 392

Jijina, J., Myers, P., \& Adams, F. 1999, ApJS, 125, 161

Jones, C., Basu, S., \& Dubinski, J. 2001, ApJ, 551, 387

Larson, R. 1969, MNRAS, 145, 271

Lin, C., Mestel, L., \& Shu, F. 1965, ApJ, 142, 1431

Mouschovias, T. 1976, ApJ, 207, 141

Myers, P., Fuller, G., Goddman, A., \& Benson, P. 1991, ApJ, 376, 551

Nakamura, F., Hanawa, T., \& Nakano, T. 1993, PASJ, 45, 551

Nakamura, F., Matsumoto, T., Hanawa, T., \& Tomisaka, K. 1999, ApJ, 510, 274

Penston, M. 1969a, MNRAS, 144, 425

Penston, M. 1969b, MNRAS, 145, 457

Ryden, B. 1996, ApJ, 471, 822

Scott, E., \& Black, J. 1980, ApJ, 239, 166

Shu, F. 1977, ApJ, 214, 488

Tafalla, M., Mardones, D., Myers, P., et al. 1998, ApJ, 504, 900

Tomisaka, K. 1991, ApJ, 376, 190

Whitworth, A., \& Summers, D. 1985, MNRAS, 214, 1 